\title{
Modelagem matemática para descrever a influência da vegetação no microclima local amazônico
}

\author{
Carlos F. L. dos Santos ${ }^{1}$ \\ UNESP, Botucatu, SP. \\ Fernando L. P. dos Santos ${ }^{2}$ \\ UNESP, Botucatu, SP.
}

\begin{abstract}
Resumo. A Amazônia brasileira tem aproximadamente 5,5 milhões de $\mathrm{km}^{2}$ de floresta e vem sendo desmatada por inúmeras razões, como aumento da área de pastagens, agricultura, construção de usinas hidrelétricas, dentre outras. O desmatamento afeta diretamente o microclima local e o convívio das populações ecológicas, colocando várias espécies em sérios riscos de extinção. Diante disso, faz-se necessários propor estratégias de recuperação das áreas degradadas. Nosso objetivo é propor um modelo matemático que descreva a recuperação da área desmatada e sua influência no microclima local. O modelo matemático é composto de um sistema não-linear de Equações Diferenciais Parciais $\left(E D P_{s}\right)$. O sistema considera fenômenos de dispersão populacional, dinâmicas vitais do tipo Verhulst e competições inter e intraespecíficas, descritas pela clássica modelagem do tipo Lotka-Volterra não- linear, combinado às $E D P_{s}$ de difusão-advecção. Os resultados numéricos, obtidos via discretização do sistema pela técnica de diferenças finitas, mostraram-se de acordo com os fenômenos considerados pelo modelo, evidenciando que as competições inter e intraespecíficas definiram a nova paisagem ecológica local, que as mudanças no uso da terra interferem diretamente no comportamento padrão do microclima local e que, portanto, as florestas tropicais são fundamentais à manutenção da estabilidade climática local.
\end{abstract}

Palavras-chave. Competições entre Espécies, Difusão-Advecção, Simulação Computacional.

\section{Introdução}

Dispersão, mortalidade de espécies, competições entre espécies na busca por sobrevivência, desmatamento, impactos ambientais e mudanças climáticas vêm sendo estudadas, analisadas e discutidas ao longo do tempo por muitos estudiosos da modelagem matemática.

A modelagem matemática em fenômenos biológicos vem sendo estudada deste Malthus (1766 1834), passando por Verhulst (1804-1849), Lotka-Volterra $(1880-1949)$ até os dias atuais.

Os modelos mencionados acima consideravam apenas a variação temporal. Neste trabalho nosso interesse principal são nas $E D P_{s}$ não-lineares, clássico no contexto da modelagem de dispersão populacional, envolvendo equação de difusão-advecção acoplados termos do tipo Lotka-Volterra e dinâmicas vitais do tipo Verhulst, [10], cuja dimensão espacial apresenta importância relevante.

A proposta é modelar matematicamente as competições pelos recursos (água, luz e nutrientes) entre três espécies de vegetação (Cumaru, Ingá, Pau-Pretinho) em uma área degradada que está em processo de recuperação, a fim de definir a futura paisagem ecológica e sua influência no microclima dessa região, neste caso, considerando na modelagem os conceitos de calor latente $(L)$

\footnotetext{
${ }^{1}$ carlos.frank@unesp.br

2 fernando.pio@unesp.br
} 
e calor sensível $(H)$ ambos medidos em $\left(W / m^{2}\right)$. O processo de reflorestamento pode impactar significativamente no clima regional e global, ocasionando aumento de 5 a $20 \%$ na precipitação (chuva) [4].

O aumento de $L$ implica diretamente em aumento de precipitação (chuva), a redução de $L$ implica diretamente em escassez de precipitação (chuva), assim como o aumento de $H$ implica diretamente em aumento de temperatura, a redução de $H$ implica diretamente em diminuição de temperatura.

Em áreas desmatadas, a maior parte da energia disponível a superfície é utilizada para aquecer o ar, devido a não disponibilidade de água para evaporar. Assim, o fluxo de $H$ aumenta e $L$ diminui. Nas áreas de floresta, $H$ representa apenas $14 \%$ utilizado para aquecimento, e $L 71 \%$ utilizado para precipitação [11].

O modelo proposto é resolvido numericamente pelo método de Diferenças Finitas Centrais de segunda ordem para a variável espacial combinado ao método de Diferenças Finitas de CrankNicolson para variável tempo. Simulações computacionais foram performadas resolvendo-se o modelo discretizado, com o intuito de visualizar a dinâmica espaço-temporal do processo de recuperação da área degradada, sob as influências climáticas locais expressas pelos fatores, como calor latente e calor sensível.

A Seção seguinte descreve o modelo matemático proposto para elucidar a dinâmica de recuperação de uma área degradada e sua influência no clima local.

\section{Modelo Matemático}

O sistema de equações diferenciais parciais dado no modelo (1) descreve a recuperação da área degrada e sua influência no microclima local em cada ponto $(x, y)$ do domínio retangular $\Omega=[a ; b] \times[a ; c] \subset \Re^{2}$, aberto, não vazio e fronteira $\partial \Omega$ suficientemente regular em cada instante de tempo $t \in(0, T]$, sendo $T$ o tempo total.

O domínio computacional bidimensional considera uma população com 48 indivíduos (plantas), sendo 16 de cada espécie $\left(P_{1}, P_{2}, P_{3}\right)$ a uma distância de 3 metros entre os indivíduos, distribuídos de forma alternada dispostos em 6 linhas e 8 colunas em todo domínio $\Omega$ da aplicação, como mostra a Figura 1.

\begin{tabular}{|l|l|l|l|l|l|}
\hline$P_{2}$ & $P_{1}$ & $P_{3}$ & $P_{2}$ & $P_{1}$ & $P_{3}$ \\
\hline$P_{1}$ & $P_{3}$ & $P_{2}$ & $P_{1}$ & $P_{3}$ & $P_{2}$ \\
\hline$P_{3}$ & $P_{2}$ & $P_{1}$ & $P_{3}$ & $P_{2}$ & $P_{3}$ \\
\hline$P_{2}$ & $P_{1}$ & $P_{3}$ & $P_{2}$ & $P_{1}$ & $P_{2}$ \\
\hline$P_{1}$ & $P_{3}$ & $P_{2}$ & $P_{1}$ & $P_{3}$ & $P_{1}$ \\
\hline$P_{3}$ & $P_{2}$ & $P_{1}$ & $P_{3}$ & $P_{2}$ & $P_{3}$ \\
\hline$P_{2}$ & $P_{1}$ & $P_{3}$ & $P_{2}$ & $P_{1}$ & $P_{2}$ \\
\hline$P_{1}$ & $P_{3}$ & $P_{2}$ & $P_{1}$ & $P_{3}$ & \\
\hline
\end{tabular}

Figura 1: Distribuição inicial de $P_{1}, P_{2}, P_{3}$ no domínio computacional $\Omega$. 


$$
\left\{\begin{array}{l}
\frac{\partial P_{1}}{\partial t}=\alpha_{1}\left(\frac{\partial^{2} P_{1}}{\partial x^{2}}+\frac{\partial^{2} P_{1}}{\partial y^{2}}\right)-u_{1} \frac{\partial P_{1}}{\partial x}-v_{1} \frac{\partial P_{1}}{\partial y}-\mu_{1} P_{1}+\lambda_{1} P_{1}\left[1-\frac{P_{1}+\gamma_{1} P_{2}+\sigma_{1} P_{3}}{K}\right] \\
\frac{\partial P_{2}}{\partial t}=\alpha_{2}\left(\frac{\partial^{2} P_{2}}{\partial x^{2}}+\frac{\partial^{2} P_{2}}{\partial y^{2}}\right)-u_{2} \frac{\partial P_{2}}{\partial x}-v_{2} \frac{\partial P_{2}}{\partial y}-\mu_{2} P_{2}+\lambda_{2} P_{2}\left[1-\frac{P_{2}+\gamma_{2} P_{1}+\sigma_{2} P_{3}}{K}\right] \\
\frac{\partial P_{3}}{\partial t}=\alpha_{3}\left(\frac{\partial^{2} P_{3}}{\partial x^{2}}+\frac{\partial^{2} P_{3}}{\partial y^{2}}\right)-u_{3} \frac{\partial P_{3}}{\partial x}-v_{3} \frac{\partial P_{3}}{\partial y}-\mu_{3} P_{3}+\lambda_{3} P_{3}\left[1-\frac{P_{3}+\gamma_{3} P_{2}+\sigma_{3} P_{1}}{K}\right] \\
\frac{\partial L}{\partial t}=\alpha_{4}\left(\frac{\partial^{2} L}{\partial x^{2}}+\frac{\partial^{2} L}{\partial y^{2}}\right)-\mu_{4} L+\lambda_{4} L\left[\frac{\beta_{1} P_{1}}{\beta_{1} P_{1}+K}+\frac{\beta_{2} P_{2}}{\beta_{2} P_{2}+K}+\frac{\beta_{3} P_{3}}{\beta_{3} P_{3}+K}\right] \\
\frac{\partial H}{\partial t}=\alpha_{5}\left(\frac{\partial^{2} H}{\partial x^{2}}+\frac{\partial^{2} H}{\partial y^{2}}\right)-\mu_{5} H-\lambda_{5} H\left[\frac{\delta_{1} P_{1}}{\delta_{1} P_{1}+K}+\frac{\delta_{2} P_{2}}{\delta_{2} P_{2}+K}+\frac{\delta_{3} P_{3}}{\delta_{3} P_{3}+K}\right] \\
\left.\frac{\partial P_{1}}{\partial \eta}\right|_{\partial \Omega_{i}}=-c_{j} P_{1} ; \\
\left.\frac{\partial P_{2}}{\partial \eta}\right|_{\partial \Omega_{i}}=-d_{j} P_{2} \\
\left.\frac{\partial P_{3}}{\partial \eta}\right|_{\partial \Omega_{i}}=-g_{j} P_{3} \\
\frac{\partial \Omega_{i}}{\partial \eta}=-n_{j} L ;
\end{array}\right.
$$

sendo as espécies Cumaru $\left(P_{1}\right)$, Ingá $\left(P_{2}\right)$, Pau-Pretinho $\left(P_{3}\right)$, Calor latente $(L)$ em $\left(W / m^{2}\right)$ e Calor sensível $(H)$ em $\left(W / m^{2}\right) ; \eta$ é um vetor normal a superfície; $c_{j}, d_{j}, g_{j}, n_{j}, m_{j}, j=1, \ldots, 4$ são as constantes de proporcionalidades adequadas as condições de contorno do tipo Robin [8].

Os coeficientes de difusão serão representados pelo parâmetros $\alpha_{i}$, os decaimentos são representados pelos parâmetros $\mu_{n}$, a taxa de crescimento e dada por $\lambda_{s}, i, n, s=1, \cdots, 5$, os coeficientes de advecção serão descritos por $\left\langle u_{k}, v_{k}\right\rangle, k=1,2,3$, os termos de competições interespecíficos e intraespecíficos são dados, respectivamente, por $\frac{\lambda_{s} * \sigma_{k}}{K}$, e $\frac{\lambda_{s}}{K}$ e uma capacidade de suporte $K$.

O modelo e o domínio foram discretizados visando uma solução por aproximação numérica pelo método de diferenças finitas de Crank-Nicolson no tempo [10] e diferenças finitas centrais na dimensão espacial, com condições de fronteira do tipo Robin [8].

A seguir tem-se os resultados numéricos obtidos pelas simulações computacionais de (1).

\section{Resultados Numéricos}

Nesta Seção apresentam-se os resultados numéricos das simulações computacionais do modelo (1) das dinâmicas das três espécies de vegetação, com o intuito de visualizar a recuperação da área degradada e a influência dessa regeneração no microclima local Amazônico. 
Para as simulações, consideram-se as competições inter e intraespecíficas em um domínio bidimensional $\Omega$ e o tempo $T=5000$ dias (aproximadamente 13 anos).

Tabela 1: Valores dos parâmetros usados nas simulações.

\begin{tabular}{ccccccccc}
\hline Par. & Valores & Par. & Valores & Par. & Valores & Par. & Valores & Unidade \\
\hline$\alpha_{1}$ & $0,0149 e^{-2}$ & $\alpha_{2}$ & $0,022 e^{-2}$ & $\alpha_{3}$ & $0,0127 e^{-2}$ & $\alpha_{4}$ & 0,011 & área/tempo \\
$\mu_{1}$ & 0,002 & $\mu_{2}$ & 0,001 & $\mu_{3}$ & 0,004 & $\mu_{4}$ & 0,001 & $h^{-1}$ \\
$u_{1}$ & 0,011 & $u_{2}$ & 0,01 & $u_{3}$ & 0,013 & $\alpha_{5}$ & 0,021 & área/tempo \\
$v_{1}$ & 0,05 & $v_{2}$ & 0,07 & $v_{3}$ & 0,072 & & & área/tempo \\
$\lambda_{1}$ & 0,31 & $\lambda_{2}$ & 0,58 & $\lambda_{3}$ & 0,22 & $\lambda_{4}$ & 0,021 & $\mathrm{n}^{\text {o real }}$ \\
$K$ & 16 & $n y$ & 8 & $d x$ & 0,0125 & $n x$ & 6 & $\mathrm{n}^{\text {o real }}$ \\
$\gamma_{1}$ & 0,003 & $\gamma_{2}$ & 0,02 & $\gamma_{3}$ & 0,005 & & & área $/$ ind. $t^{2}$ \\
$\sigma_{1}$ & $0,011 e^{-3}$ & $\sigma_{2}$ & $0,010 e^{-3}$ & $\sigma_{3}$ & $0,0015 e^{-3}$ & & & área $/$ ind. $t^{2}$ \\
$\beta_{1}$ & 0,001 & $\beta_{2}$ & 0,003 & $\beta_{3}$ & 0,005 & $\lambda_{5}$ & 0,013 & $\mathrm{n}^{\text {o real }}$ \\
$\delta_{1}$ & 0,002 & $\delta_{2}$ & 0,004 & $\delta_{3}$ & 0,001 & & & $\mathrm{n}^{\text {o real }}$ \\
$d t$ & 0,01 & $d y$ & 0,0125 & & & & & $\mathrm{n}^{\text {o real }}$ \\
& & & & & & $\mu_{5}$ & 0,002 & $h^{-1}$ \\
\hline
\end{tabular}

A Tabela 1 mostra os valores dos parâmetros utilizados nas simulações computacionais.

Os parâmetros de difusão foram considerados de acordo com [5], [6], [9] os de advecção foram considerados de acordo com [7], os de decaimento e taxa de crescimento foram obtidos conforme [2].

Os parâmetros de competições inter e intraespecíficas foram baseados em [1].

Inicialmente as espécies $P_{1}, P_{2}$ e $P_{3}$ tinham densidades iguais $1, L=6 \mathrm{~W} / \mathrm{m}^{2}$ e $H=8 \mathrm{~W} / \mathrm{m}^{2}$. As simulações foram feitas em ambiente Scilab 6.1, em notebook, modelo HP 240 G3, processador Intel CORE $i 3$, RAM 4 GB, com tempo médio de processamento de 47 minutos.

\subsection{Resultados referentes ao espaço}

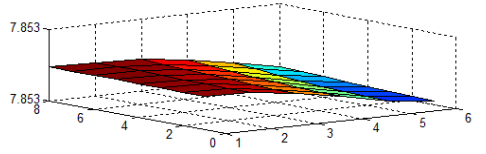

(a) Distribuição espacial final de $P_{1}$

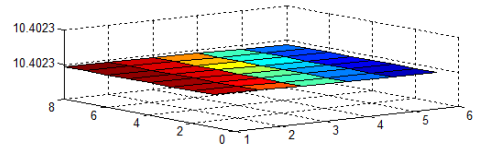

(d) Distribuição espacial final de $L$

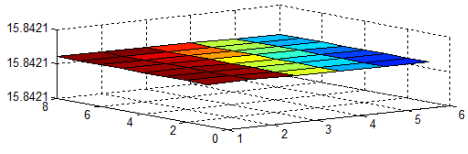

(b) Distribuição espacial final de $P_{2}$

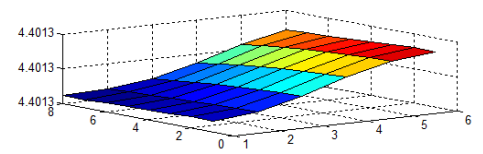

(c) Distribuição espacial final de $P_{3}$

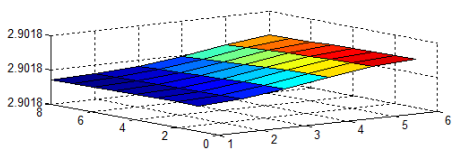

(e) Distribuição espacial final de $H$

Figura 2: Resultados numéricos computacionais das dinâmicas espaciais de $P_{1}, P_{2}, P_{3}, L$ e $H ; \Delta x=$ $\Delta y=0,0125 ; \Delta t=0,01 ; T=13$ anos. Cores próximas do azul indicam baixa densidade da espécie e próximo ao vermelho, alta densidade da espécie.

A sequência de Figuras 2(a) a 2(e) descrevem a partir das condições iniciais as dinâmicas espaciais de cada espécie de vegetação e o comportamento espacial de $L$ e $H$ descrito pelo modelo (1). 
- A Figura 2(a) descreve os efeitos dos fenômenos considerados no modelo (1) sobre a espécie $P_{1}$. Podemos observar o espalhamento natural de $P_{1}$ por todo o domínio, devido os efeitos da difusão e da advecção provocando a sobreposição de nicho acarretando as competições e inibindo o crescimento natural de espécies com baixo poder de competição. Ainda nesta Figura, é possível notar uma região do domínio em que a densidade de $P_{1}$ é menor que em outra. Isso pode ser explicado pela intensificação das competições entre as três espécies.

- A Figura 2(b) mostra a dinâmica espacial da espécie $P_{2}$. Pode-se notar nesta dinâmica que as competições não provocaram inibição de crescimento nesta espécie. Uma explicação que pode ser dada é que a espécie $P_{2}$, na sobreposição de nicho, pode ter desenvolvido melhor estratégia de competição dificultando acesso das demais espécies aos recursos disponíveis, inibindo o crescimento das demais espécies.

- A Figura 2(c) exibe a dinâmica espacial da espécie $P_{3}$. Nota-se neste resultado que os efeitos mais evidentes das competições provocadas pela sobreposição de nicho (devido difusão e adveç̧̃a) que inibiu o crescimento dessa espécie. A baixa densidade dessa população poderia ser explicada por $P_{3}$ ter tido alguma desvantagem na competição com as demais espécies, obtendo menos recursos para o seu desenvolvimento. Em regiões distintas do domínio $\Omega$ esta espécie teve densidades diferentes, na região de maior densidade ocorre exatamente onde $P_{1}$ tem menor densidade, isso decorre das competições com $P_{1}$ ter sido favorável a $P_{3}$ nesta região;

- A Figura 2(d) descreve o comportamento do calor latente, $L$, que se manteve estável, isso ocorre devido a influência da vegetação nas bordas que propagam $L$ para todo domínio por meio da difusão. Como a região já está sendo coberta por vegetação, então $L$ começa a ser predominante também nessa região;

- A Figura 2(e) descreve o comportamento do calor sensível, $H$. Aqui é possível observarmos uma intensidade maior de $H$ na parte em que as espécies $P_{1}$ e $P_{2}$ tem densidade mais baixa e o calor latente $L$ tem menor concentração. Como antes a região era desmatada, isso contribuiu para grande concentração de $H$ nessa região e aos poucos essa concentração está se desfazendo devido a influência da vegetação que está se recuperando na região e aumentando a densidade do calor latente $L$.

Em síntese, a densidade final de cada espécie foi: $P_{1}=7,85, P_{2}=15,84, P_{3}=4,4, L=10,4$ e $H=2,9$. Comparando-se com os valores inicias de $\mathrm{L}$ e $\mathrm{H}$, houve $73,3 \%$ de aumento no calor latente e 51,6\% de redução no calor sensível. Na Figura 2 podemos observar que a espécie $P_{2}$ predominou em todo domínio $\Omega$ definindo a nova paisagem ecológica.

\subsection{Resultados referentes ao tempo}

A Figura 3 descreve as dinâmicas temporais das três espécies $P_{1}, P_{2}$ e $P_{3}$ e sua influência no microclima local ao decorrer do tempo e ao longo do domínio computacional estabelecido. 


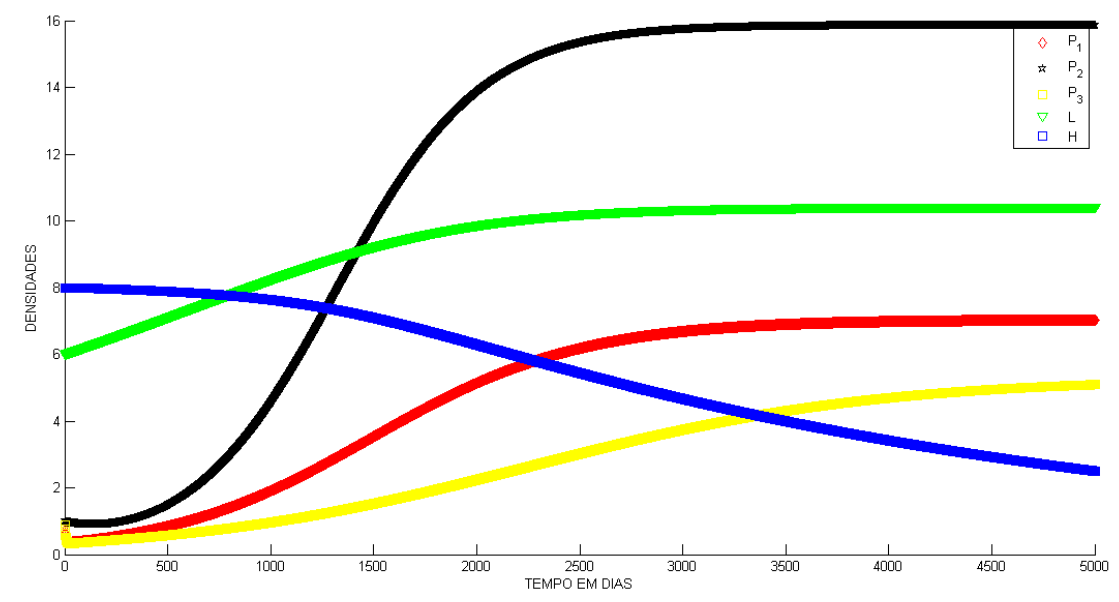

Figura 3: Resultados numéricos computacionais das dinâmicas temporais de $P_{1}, P_{2}, P_{3}, L$ e $H, \Delta t=0,01$; $T=5000$ dias.

A Figura 3 mostra que com o passar do tempo há uma estabilidade entre as espécies e que em aproximadamente 13 anos a área antes degradada estará recuperada com a predominância da espécie $P_{2}$, definindo a nova paisagem ecológica no meio desmatado.

Os resultados mostram ainda que em áreas desmatadas, $H$ é predominante em relação a $L$. Constata-se que a medida que a densidade de vegetação vai se recuperando na região desmatada, ocorre uma inversão de fluxo de calor, ou seja, $H$, que era predominante, se reduz e $L$, que era menos intensa, passa a predominar na região onde antes era dominado por $H$. Isso é uma clara manifestação da influência da vegetação no microclima.

A Seção seguinte sintetiza as principais conclusões acerca dessa investigação numérica computacional dos efeitos da vegetação no clima local.

\section{Conclusões}

Há evidências de resultados extremamente prejudiciais à vida e a biodiversidade a curto, médio e longo prazos decorrentes de uma larga gama de efeitos de impactos ambientais. Dentre estes o desmatamento, a redução da precipitação e o aumento da temperatura, afetam de modo irreversível o delicado e instável equilíbrio do convívio de espécies que se inter-relacionam ambientalmente.

O objetivo deste estudo foi propor um modelo matemático que descreva a recuperação da área desmatada considerando competições de modo intra e interespecíficas das espécies $P_{1}, P_{2}$ e $P_{3}$, elucidando futura sucessão ecológica e sua influência no microclima local.

A partir dos resultados das simulações, concluí-se o seguinte:

- O aumento da difusão provoca forte influência na ocorrência das competições, que por sua vez define a nova paisagem ecológica;

- O fenômeno advectivo é um fator determinante na coexistências de algumas espécies e formação de floresta mista;

- A nova paisagem ecológica somente pode ser visualizada no domínio quando se considera os efeitos difusivo e advectivo na modelagem; 
- Essa nova paisagem ecológica está sendo definida pela espécie $P_{2}$ que desenvolve melhor estratégias de competição na captação dos recursos;

- A cobertura vegetal em áreas desprovidas de vegetação pode provocar mudanças no fluxo de energia ocasionando redução da temperatura e aumento na precipitação.

De modo geral, a mudança do solo desprovido de vegetação para o solo com a cobertura vegetal devido o reflorestamento afeta diretamente os fluxos de calor latente $(L)$ e calor sensível $(H)$. Além disso, como o aumento de $L$ implica no aumento da precipitação e a redução de $H$ acarreta em diminuição da temperatura [3], se torna evidente que as mudanças no uso da terra interferem diretamente no comportamento padrão do microclima local e que as florestas tropicais são fundamentais à manutenção da estabilidade climática local.

\section{Referências}

[1] Gotelli, N. J., Graves, G. R. Null models in ecology, 2a. edição. Princeton Editorial, London, 1996.

[2] Jaquetti, R. K. Ecophysiology of fabaceae tree species during forest restoration in the balbina hydroeletric dam in Amazonas state, Tese de Doutorado, Instituto Nacional de Pesquisas da Amazônia, 2018.

[3] Lyra, R., Noble, C., Fisch, G., Rocha, E., Rocha, H. e Souza, S. Efeitos do desmatamento na termodinâmica da baixa atmosfera Anais do VIII Congresso Meteorológico Brasileiro, volume 1:81-84, 1994.

[4] Malhi, Y. The carbon balance of tropical forest regions, 1990-2005. Current Opinion in Environmental Sustainability, 2:237-244, 2010.

[5] Marchuk, G. I. Mathematical models in environmental problems, 16a. edição. North Holland, 1986.

[6] Okubo, A. Diffusion and Ecological Problems: Mathematical Models, 1a. edição. Springer, 1980.

[7] Prestes, M. F. B. Dispersão de material impactante em meio aquático: Modelo matemático, aproximação numérica e simulação computacional-Lagoa do Taquaral, Dissertação de Mestrado, Unicamp, 2011.

[8] Santos, C. F. L. Modelagem matemática do aumento de densidade de vegetação na Amazônia e dinâmica populacional com competição intra e interespecífica, Dissertação de Mestrado, Unicamp, 2013.

[9] Skellam, J. C. Random dispersal in theoretical populations, 38a. edição. Biometrika, London, 1951.

[10] Sossae, R. C. A presença evolutiva de um material impactante e seu efeito no transiente populacional de espécies interativas: modelagem e aproximação, Tese de Doutorado, Unicamp, 2003.

[11] Souza, S. S. A substitução da floresta amazônica por pastagem e sua repercussão ao nível da termodinâmica da camada limite atmosférica. Dissertação de Mestrado, Universidade Federal de Campina Grande, 1997. 